\title{
BOUNDEDNESS OF LIMITS ${ }^{1}$
}

\section{CHIN-SHUI HSÜ}

This is an answer to a problem of A. Wilansky [2]. Suppose $\sum_{1}^{\infty} b_{k}$ converges absolutely. Out of each infinite sequence $x=\left\{x_{n}\right\}$ we may construct

$$
\beta_{1}(x)=x_{1}, \quad \beta_{n}(x)=x_{n}+x_{n-1}+\sum_{1}^{n-1} b_{k} x_{k} \quad(n>1) .
$$

Obviously $\|x\|=1$.u.b. $\left\{\left|\beta_{1}(x)\right|,\left|\beta_{2}(x)\right|, \cdots\right\}$ forms a norm over the linear space of all convergent sequences. The problem is whether the linear functional $\lim x=\lim _{n \rightarrow \infty} x_{n}$ is bounded in this norm.

We may solve (1) to have

$$
x_{n}=\sum_{r=1}^{n}(-1)^{n-r} g_{n}^{(r)} \beta_{r}(x)
$$

where

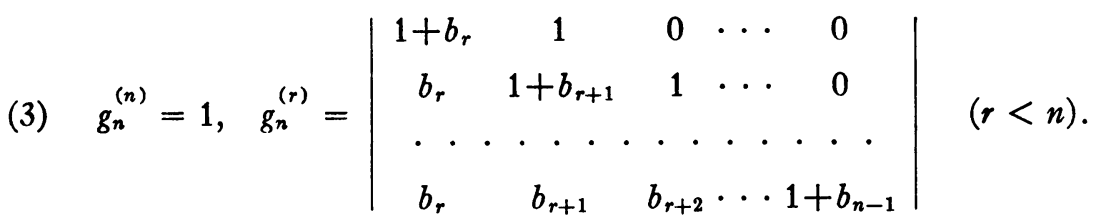

On expanding $g_{n}^{(r)}$ along the diagonal we have $g_{n}^{(r)}=1+$ linear combination of products of $b_{r}, \cdots, b_{n-1}$ with factors of $\left(1-b_{r}\right)\left(1-b_{r+1}\right)$ $\cdots\left(1-b_{n-1}\right)$ as coefficients. Hence

(4) $\quad\left|g_{n}^{(r)}\right|<B, \quad\left|g_{n}^{(r)}-1\right|<C\left(\left|b_{r}\right|+\cdots+\left|b_{n-1}\right|\right)$

where $B, C$ are independent of $n$ and $r$.

By (3) we also have

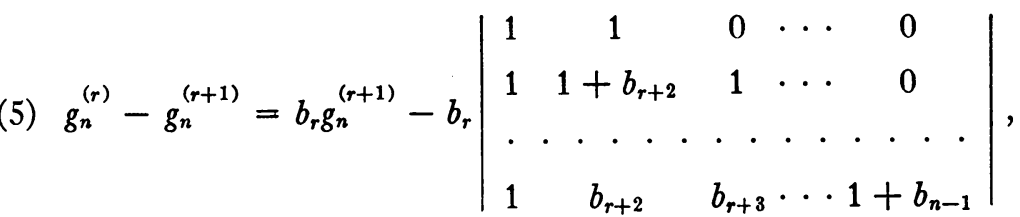

Received by the editors October 13, 1961.

${ }^{1}$ Editorial note: After this paper had been accepted for publication a specific counterexample to Wilansky's conjecture was published by Hayman and Wilansky[1]. 


$$
\begin{aligned}
g_{n}^{(r)}-g_{n-1}^{(r)}= & b_{n-1} g_{n-1}^{(r)}-b_{n-2} g_{n-2}^{(r)}+\cdots+(-1)^{n-r} b_{r+1} g_{r+1}^{(r)} \\
& +(-1)^{n-r-1} b_{r} .
\end{aligned}
$$

Expanding (5) along the diagonal and arguing as in (4),

$$
\left|g_{n}^{(r)}-g_{n}^{(r+1)}\right|<D\left|b_{r}\right|
$$

where $D$ is independent of $n$ and $r$.

Let

$$
z_{1}=x_{1}, \quad z_{n}=x_{n}+x_{n-1}, \quad \beta_{n, r}=\beta_{r}-\beta_{r+1}+\cdots+(-1)^{n-r} \beta_{n},
$$

then

$$
z_{n}=k_{n}^{(1)} \beta_{1}(x)+\cdots+k_{n}^{(n-1)} \beta_{n-1}(x)+\beta_{n}(x)
$$

where

$$
\begin{aligned}
k_{n}^{(r)}= & (-1)^{n-r} g_{n}^{(r)}+(-1)^{n-r-1} g_{n-1}^{(r)}=-b_{r}+b_{r+1} g_{r+1}^{(r)}-\cdots \\
& +(-1)^{n-r} b_{n-1} g_{n-1}^{(r)} .
\end{aligned}
$$

Since $\beta_{r+2 s+1, r}=x_{r-1}-x_{r+2 s+1}-\left(b_{r} x_{r}+\cdots+b_{r+2 s} x_{r+2 s}\right)$, we have

$$
\left|\beta_{n, r}\right|<E
$$

where $E$ is independent of $n$ and $r$. Then

$$
\begin{aligned}
\left|\sum_{r=s+1}^{n-1} k_{n}^{(r)} \beta_{r}(x)\right| & =\left|k_{n}^{(s+1)} \beta_{n, s+1}+\sum_{r=8+1}^{n-2}\left(k_{n}^{(r)}+k_{n}^{(r+1)}\right) \beta_{n, r}+k_{n}^{(n-1)} \beta_{n, n-1}\right| \\
& \leqq E\left\{(2 C+2 D)\left(\left|b_{s+1}\right|+\cdots+\left|b_{n-1}\right|\right)\right. \\
& \left.+2 C\left|b_{n-1}\right|\right\} .
\end{aligned}
$$

Hence, from (8),

$$
\lim x=\frac{1}{2}\left[\lim _{n \rightarrow \infty} \beta_{n}(x)+\sum_{1}^{\infty} k^{(r)} \beta_{r}(x)\right]
$$

where

$$
k^{(r)}=\lim _{n \rightarrow \infty} k_{n}^{(r)}=\sum_{s=0}^{\infty}(-1)^{s-1} g_{r+s}^{(r)} b_{r+s .}
$$

THEOREM. The linear functional lim is bounded if and only if $\sum_{1}^{\infty}\left|k^{(r)}\right|<\infty$.

Proof. If $\sum_{1}^{\infty}\left|k^{(r)}\right|<\infty$, then (11) implies

$$
|\lim x|<\frac{1}{2}\left(1+\sum_{1}^{\infty}\left|k^{(r)}\right|\right)\|x\| \text {. }
$$


If $\sum_{1}^{\infty}\left|k^{(r)}\right|=\infty$, then for any positive number $M$ we have $\left|k^{(1)}\right|+\left|k^{(2)}\right|+\cdots+\left|k^{(m)}\right|=K>4 M$ for some $m$ large enough. Let $\beta_{i}=\left(2 M\left|k^{(i)}\right|\right) /\left(K k^{(i)}\right)$ for $k^{(i)} \neq 0$ and $i \leqq m, \beta_{i}=0$ for $k^{(i)}=0$ or $i>m$. Construct $x_{n}$ and $z_{n}$ according to (2) and (8), then $\lim _{n \rightarrow \infty} z_{n}$ $=2 M$ and by (9) whenever $n>m,\left|z_{n}-z_{n+1}\right|<(m B)\left|b_{n}\right|$. So $\left(z_{2}-z_{1}\right)$ $+\left(z_{4}-z_{3}\right)+\cdots$ converges absolutely. Hence

$$
\begin{aligned}
x_{2 n} & =\left(z_{2}-z_{1}\right)+\left(z_{4}-z_{3}\right)+\cdots+\left(z_{2 n}-z_{2 n-1}\right) \rightarrow M+\xi, \\
x_{2 n+1} & =z_{2 n+1}-x_{2 n} \rightarrow M-\xi .
\end{aligned}
$$

(i) $\xi=0$. Then $\lim x=M$ and $\|x\|<1$.

(ii) $\xi \neq 0$. There is an integer $p>m$ such that $\left|b_{p+1}\right|+\left|b_{p+2}\right|+\cdots$ $<1 / 2|\xi|$. Let

$$
\begin{array}{lr}
x_{n}^{\prime}=x_{n} & (n \leqq p), \\
x_{n}^{\prime}=x_{n}+\xi & (n>p \text { is odd }), \\
x_{n}{ }^{\prime}=x_{n}-\xi & (n>p \text { is even }) .
\end{array}
$$

Then $\lim x^{\prime}=M$ and $\left\|x^{\prime}\right\|<1$.

Hence lim is unbounded.

REMARK. There are cases for both possibilities. Suppose $\left|b_{k}\right|$ $=O\left(k^{-\alpha}\right), \alpha>3 / 2$. It is not difficult to verify (using the second inequality of (4)) that $\left|k^{(r)}\right|=\left|b_{r}-b_{r+1}+\cdots\right|+O\left(r^{-2(\alpha-1)}\right)$. Then $\sum_{r=1}^{\infty}\left|k^{(r)}\right|$ converges or diverges with $\sum_{r=1}^{\infty}\left|b_{r}-b_{r+1}+\cdots\right|$.

\section{REFERENCES}

1. W. Hayman and A. Wilansky, An example in summability, Bull. Amer. Math. Soc. 67 (1961), 554-555.

2. A. Wilansky, An elementary inequality, Bull. Amer. Math. Soc. 67 (1961), 355.

UNIVERSITY OF HONG KoNG 